\title{
Power transistor-assisted Sen transformer: a novel approach to power flow control
}

\begin{abstract}
The importance of flexible power flow control in electric power transmission networks is increasing owing to many factors that have arisen. Sen Transformer (ST) is one of the attractive power flow controllers. It has a wide control range, independent active and reactive power flow control capability, and is economically attractive. However, it operates in stepwise mode, has limited operating points, relatively slow response rate, and suffers from compensation error. Motivated by the limitations of the ST, power transistor assisted ST (TAST) as a novel power flow controller is proposed in this paper. The TAST consists of a highly-rated ST and a lowly-rated AC chopper based transistorized ST (TST). This paper first points to the importance of transmission linesô power flow control and reviews available power flow control devices. It then introduces the proposed TAST, determines the ratings of the TST and the switching pattern of its choppers. Next, it demonstrates the steady-state performance of the TAST, determines its control limits, compares the TAST to the ST and the unified power flow controller (UPFC), and the different control strategies of the TAST. In this paper, the TAST is modeled in MATLAB/SIMULINK and tested in an equivalent two bus system and in the IEEE-14 bus test system. The work also demonstrates improvement of the response rate of the ST. Cost analysis of the TAST is also done and is compared to that of the equivalent UPFC. Based on the results, the TAST realizes continuous, error-free, more flexible operation, improved response rate, and low cost. Moreover, the TAST provides $14.62 \%$ wider power flow control range. In conclusion, the TAST's operational characteristics are closely comparable to that of the UPFC with the advantage of lower cost and extended control area.
\end{abstract}

Keyword: AC voltage regulator; FACTS; Power flow control; PWM; ST; UPFC 